\title{
A vallecular cyst in a patient with deep neck infection causing difficult airway management
}

\author{
Hyun-Min Bae, Jun-Ro Yoon, Joo-Hyun Yoo, Yun-Joung Han, and Yoo-Jung Park \\ Department of Anesthesiology and Pain Medicine, College of Medicine, The Catholic University of Korea, Seoul, Korea
}

Vallecular cyst is a very rare lesion. Vallecular cyst results from obstruction and retention of mucus in collecting ducts of submucosal glands [1]. Vallecular cyst can be potentially dangerous during anesthesia because they may completely obstruct the larynx or the anesthesiologist's view of the larynx causing difficult intubation and thus become life-threatening $[2,3]$.

We recently encountered a patient with a vallecular cyst which caused near-lethal airway obstruction during the induction. The patient was scheduled to have an urgent debridement on diagnosis of septic shock, due to deep neck infection. After preoxygenation, the patient was induced with thiopental sodium and succinylcholine acetate IV. Inserted direct laryngoscopy revealed a cyst arising from the vallecula, overlying the lingual surface of the epiglottis. The cyst hanging down perpendicular to the posterior pharyngeal wall, completely prevented visualization of the glottic opening. Furthermore, it was not possible to expose the laryngeal inlet by the laryngoscope. Sedation and muscle relaxation likely propelled the cyst further downward, due to extension with the force of gravity. Three attempts at blind intubation with a styletted $7.0 \mathrm{~mm}$ endotracheal tube, by two anesthesiologists, were unsuccessful due to the drooping cyst. Moreover, effectively bag-ventilating the patient was impossible. Meanwhile, bradyarrhythmia (40 to $45 \mathrm{bpm}$ ) and subsequently ventricular tachycardia up to $160 \mathrm{bpm}$ appeared on electrocardiograph. We clinically made a decision that further attempts at intubation were futile, on considering the fatal vital signs. Tracheostomy was immediately performed and completed within 3 minutes. After ventilation was resumed, vital signs stabilized. The surgeon intra-orally incised the cyst with base attached to the valleculae. The contents, consisting of $6 \mathrm{ml}$ of a thick, yellow purulent material were drained. The pathology report of the surgical specimen indicated a stratified squamous epithelial lined cyst, containing inflammatory debris. Review of the preoperative cervical CT indicated the presence of vallecular cyst, which had been overlooked by both the otolaryngologists and the surgical team preoperatively (Fig. 1).
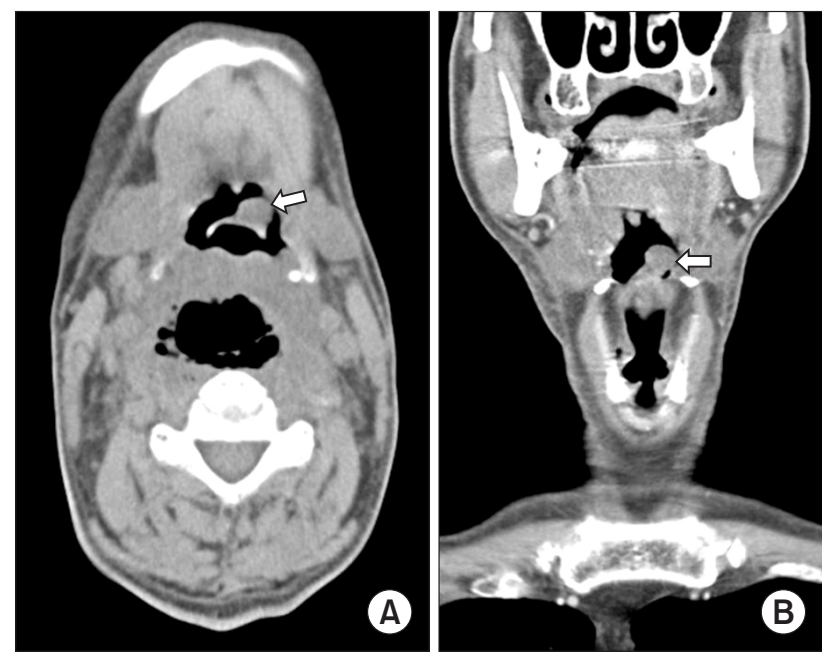

Fig. 1. (A) Computed tomographic enhanced transverse image of vallecular cyst. It shows the vallecular cyst (arrow) measuring $1.3 \times 1.1$ $\mathrm{cm}$ with distorted epiglottis. (B) Sagittal image illustrating the cyst. A hypodense mass (arrow) was visible at the top of the epiglottis.

Corresponding author: Jun-Ro Yoon, M.D., Ph.D., Department of Anesthesiology and Pain Medicine, Bucheon St. Mary's Hospital, College of Medicine, The Catholic University of Korea, 327, Sosa-ro, Wonmi-gu, Bucheon 420-717, Korea. Tel: 82-32-340-7075, Fax: 82-32-340-2255, E-mail: pauly@catholic.ac.kr

(c) This is an open-access article distributed under the terms of the Creative Commons Attribution Non-Commercial License (http:// creativecommons.org/licenses/by-nc/3.0/), which permits unrestricted non-commercial use, distribution, and reproduction in any medium, provided the original work is properly cited. 
The anesthetic implications for vallecular cyst are as follows: Firstly, there are a variety of airway rescue methods with anecdotal recommendations. Previous case reports proposed laryngeal mask airway, esophageal-tracheal combitube, blind intubation with the aid of a variety of stylets, a lightwand, transtracheal jet ventilation and tracheostomy in cases of vallecular cyst [2-4]. Secondly, there is a possibility of connection between vallecular cyst and comorbid diseases. Vallecular cyst is known to be associated with supraglottitis, epiglottitis, or epiglottic abscess $[1,5]$. Preoperative awareness by the anesthesiologist of the possibility of coexistence of a vallecular cyst in cases of deep neck infection, is important. Finally, recognizing the preanesthetic diagnostic image of vallecular cyst is essential to prevent deleterious consequences. Ultra-conservative preoperative evaluation of laryngeal structure should be made by thorough investigation when managing patients with a propensity for vallecular cyst, including deep neck infection. This case highlights the obligatory responsibility of anesthesiologists to be familiar with interpretation of airway structure images. We emphasize that early diagnosis and appropriate management of the vallecular cyst could prevent unfavorable clinical outcomes.

\section{References}

1. Berger G, Averbuch E, Zilka K, Berger R, Ophir D. Adult vallecular cyst: thirteen-year experience. Otolaryngol Head Neck Surg 2008; 138: 321-7.

2. Kamble VA, Lilly RB, Gross JB. Unanticipated difficult intubation as a result of an asymptomatic vallecular cyst. Anesthesiology 1999; 91: 872-3.

3. Rivo J, Matot I. Asymptomatic vallecular cyst: airway management considerations. J Clin Anesth 2001; 13: 383-6.

4. Dob DP, McLure HA, Soni N. Failed intubation and emergency percutaneous tracheostomy. Anaesthesia 1998; 53: 72-4.

5. Henderson LT, Denneny JC 3rd, Teichgraeber J. Airway-obstructing epiglottic cyst. Ann Otol Rhinol Laryngol 1985; 94: 473-6. 